ISSN: 2637-885X

\title{
Hirayama Disease: A Report of Two Cases and Review of Imaging Findings
}

\author{
Valentina Munera Orozco'; Angela Maria Restrepo Gil2; Jose Luis Ascencio Lancheros²; Alejandro Londoño Mesa ; Yensa \\ Rodríguez Alvarez ${ }^{*}$ \\ ${ }^{1}$ Department of Radiology, Universidad Pontificia Bolivariana Medellín, Antioquia, Colombia \\ ${ }^{2}$ Department of Radiology, Instituto Neurológico de Colombia, Medellín, Antioquia, Colombia \\ ${ }^{3}$ Department of Physical Medicine and Rehabilitation, Hospital San Vicente Fundación, Medellín, Antioquia, Colombia \\ ${ }^{4}$ Department of Radiology, Hospital Universitari Mútua Terrassa, Barcelona, Spain
}

\section{*Corresponding Author(s): Yensa Rodríguez}

Alvarez

Department of Radiology, Hospital Universitari

Mútua Terrassa, Barcelona, Spain

Fax: 665771619, Tel: +34628572778;

Email: yera22 @hotmail.com

Received: May 01, 2020

Accepted: Jun 05, 2020

Published Online: Jun 09, 2020

Journal: Journal of Radiology and Medical Imaging

Publisher: MedDocs Publishers LLC

Online edition: http://meddocsonline.org/

Copyright: (C) Rodríguez Alvarez Y (2020). This Article is

distributed under the terms of Creative Commons

Attribution 4.0 International License

\section{Abstract}

Hirayama disease is a rare self-limiting cervical myelopathy characterized by juvenile muscular atrophy of the distal upper extremities. Hirayama disease was first reported in the eastern world literature, including Asia and India. However, reports from outside of these geographic regions are increasing. Therefore, clinicians and radiologists should be aware of and familiarize themselves with the clinical features and imaging findings of this disease. We report the cases of two young Colombian males: the first individual had an atypical course affecting both upper limbs; the second male had numbness after several cervical traumas. Both individuals had Hirayama disease based on MRI (Magnetic Resonance Imaging) criteria. MRI scan in neutral position revealed atrophy and gliosis in the anterior horn of the lower cervical spine, and MRI scan with neck flexion showed anterior displacement of the posterior dura in both cases.

Keywords: Hirayama disease; Juvenile muscular atrophy; Segmental spinal muscular atrophy; Brachial monomelic amyotrophy; Cervical myelopathy.

Cite this article: Múnera Orozco V, Restrepo Gil AM, Ascensio Lancheros JL, Londoño Mesa A, Rodríguez Alvarez Y. Hirayama Disease: A Report of Two Cases and Review of Imaging Findings. J Radiol Med Imaging. 2020: 3(1); 1030. 


\section{Introduction}

Hirayama Disease (HD) is a non-progressive cervical myelopathy that differs from motor neuron disease. It was first described by Keizo Hirayama in 1959 [1]. The disease is prevalent in male individuals ranging from 15 to 25 years of age. Males are more affected than females [2]. Common clinical characteristics of HD include insidious onset of unilateral or asymmetric wasting and weakness of muscles in $\mathrm{C} 7$ to $\mathrm{T} 1$ myotomes, with a relative sparing of the brachioradialis muscle, followed by spontaneous arrest within 3 to 5 years [3].

It has been considered a benign and self-limited disease. However, the disease may have atypical characteristics, such as, progressive and irreversible course with poor prognosis, impairing the patient's quality of life $[2,4]$.

Dynamic MRI of the cervical spine (neutral and flexion position) is the imaging modality of choice for the diagnosis of HD $[2,3,5]$. Several cases of HD remain undetected, probably as a result of a lack of diagnostic criteria. In this report we describe the most remarkable findings observed during MRI in patients with HD, all these findings, are illustrated in two cases diagnosed in Colombia, South America.

\section{Case 1}

A 13 year-old man presented with one-year progression of sudden loss of strength and atrophy of upper limb, compromising the right forearm and hand.

The physical examination revealed asymmetry of the upper limbs and atrophy of forearms and hands, predominantly in the right side (Figure 1a). No alterations were observed on the cranial nerves, nor on reflexes and sensitivity. Deep reflexes were normal $(++)$, except for a decreasing in styloradial reflexes $(+)$. A brain MRI was conducted, and no pathological findings were observed.

A small decreasing of the anteroposterior diameter of the spinal cord at $\mathrm{C} 5$ and $\mathrm{C} 6$ was found through MRI of the cervical spine in neutral position. Bilateral hyperintensities of the anterior horns with a multilevel and asymmetrical compromise from C5 to C7 was also found. Greater compromise in C5-C6 was observed due to focal myelomalacia as shown by the 'Snake Eye' sign (Figure 1b). T1 and T2 sequence of the cervical spine in neutral and flexion position were conducted. On T1 with neck flexion duramater was shown to be only slightly deatached from the posterior lamina, with an increasing of the laminodural space (LDS) at C5-C6 of about $4.2 \mathrm{~mm}$ (Figure 1c).
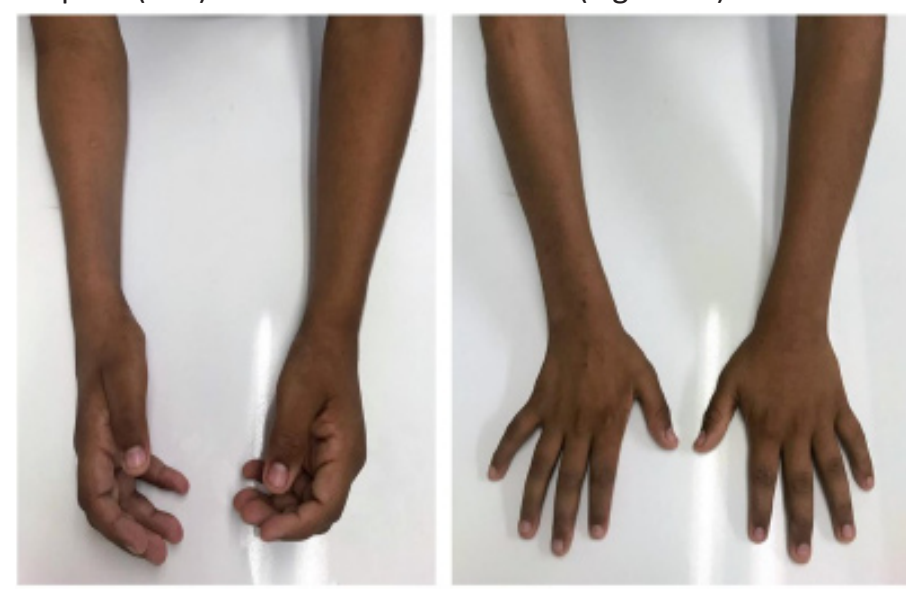

Figure 1a: 13-year-old male during physical exam presenting forearms and hands atrophy, predominantly in the right side.
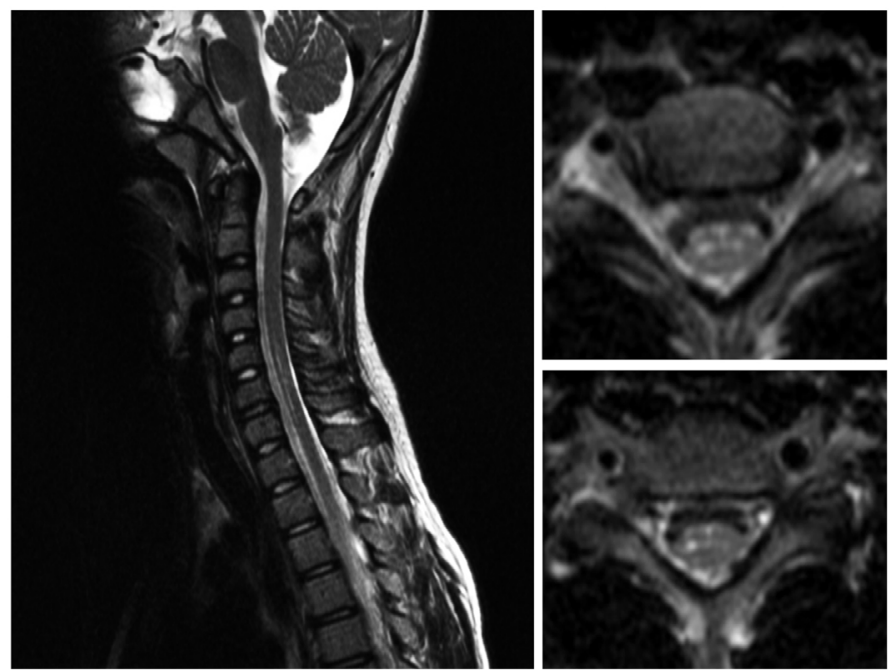

Figure 1b: Neutral-position MR images in a 13-year-old male patient with sudden loss of strength and atrophy, which is greater on the right side than on the left.

-Sagittal T2-weighted image shows flattening and faint non compressed intramedullary high signal intensity at C5 to C7.

-Transverse T2 weighted MR image at pedicular level of C5 and C6 shows the typical snake eyes sign that is characterized as a small high-signal-intensity of the bilateral anterior horns, in this case asymmetric predominantly in the right side.
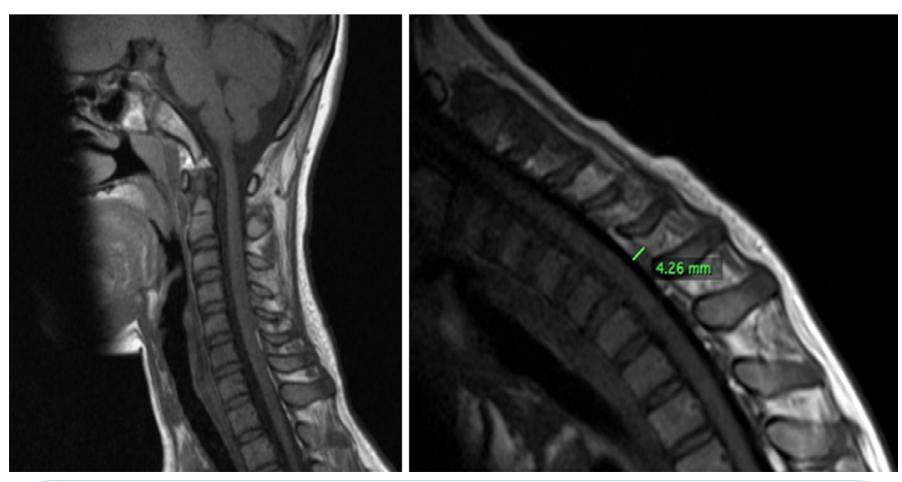

Figure 1c: -Neutral position sagittal T1-weighted image shows the normal appearance of the cervical cord

-Sagittal T1-weighted flexion MR images widening of the LDS with anterior displacement of the posterior dura in $4.26 \mathrm{~mm}$.

\section{Case 2}

A 13 year-old man presented with a background of moderate cervical trauma, which occurred twice before evaluation. Patient came due to a cervicalgia associated to dysesthesia on the lateral sides of the fingers in both hands and sensitivity alteration in the back of the limbs when flexion of the neck was forced. The physical exam revealed hyperreflexia of the four limbs, predominantly in left side, and symptoms of cervical instability with positive Lhermitte's sign.

A decrease in volume of the cervical spinal cord with subtle hyperintensity at C5-C6 was observed through an MRI of the cervical spine during neutral and flexed position (Figure 2a). Likewise, an increase of the LDS of about $6 \mathrm{~mm}$ with a decrease of the amplitude of the canal was observed in the T2 sagittal image during flexion.

Figure $2 \mathrm{~b}$ shows that dura mater has migrated and disinserted from the posterior dural sac (up to $6 \mathrm{~mm}$ ). In addition, 
engorgement of the posterior epidural venous plexus with displacement of the spinal cord contacting the posterior wall of the vertebral body was observed.
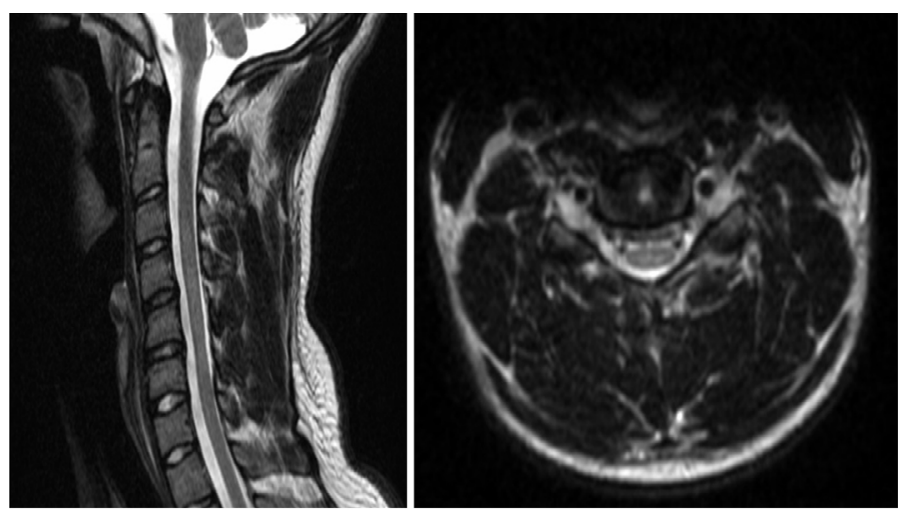

Figure 2a: Neutral-position MR images in a 17-year-old male patient with dysesthesia on the lateral sides of the fingers in both hands and sensitivity alteration in the back of the limbs when flexion of the neck was forced. A Sagital and axial T2-weithed images in neutral position demonstrates cord atrophy and abnormal cord signal at C5-C6. There is loss of the normal cervical spine lordosis.

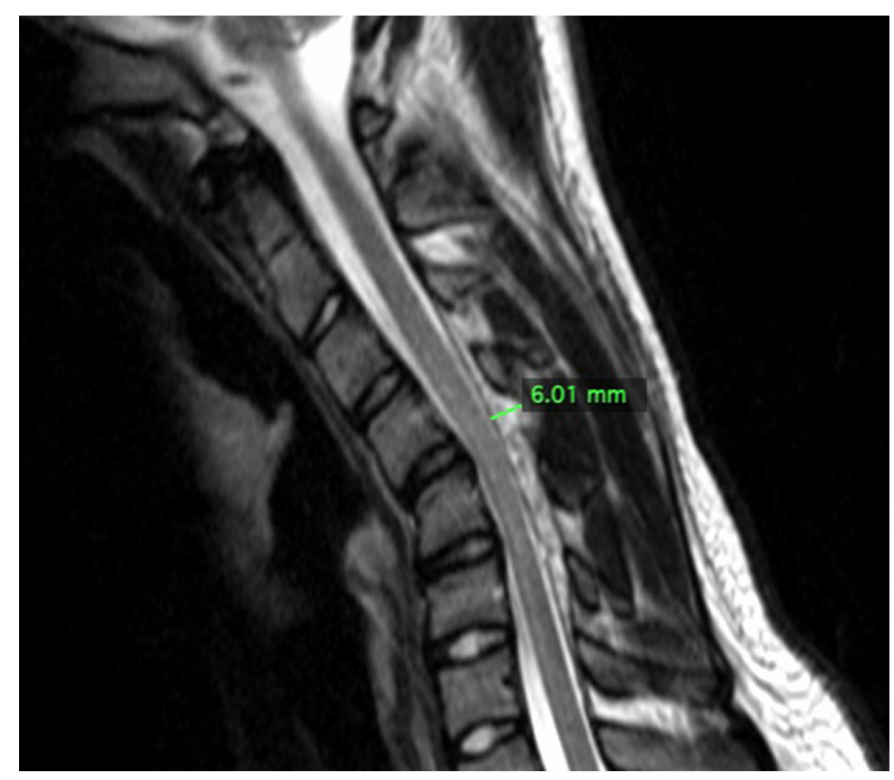

Figure 2b: Sagittal T2-weighted flexion MR images show forward displa cement of the posterior dural wall, more prominent at C5, with widening of the posterior epidural space which contains flow voids. Widening of the LDS was $6 \mathrm{~mm}$.

\section{Discussion}

The literature describes several clinical criteria for HD, including onset of symptoms in late adolescence or young adulthood, restricted motor function in the upper limbs, asymmetrical muscular changes, a period of clinical progression following a relative stable condition, and the non-existence of alternative assessments for symptoms identified during evaluation [6].

$M R I$ in neutral and flexed positions are required for the diagnosis of $\mathrm{HD}[2,3,5]$. The following findings in MRI are correlated with the etiopathogenesis of the disease.

-Inferior cervical spinal cord atrophy: Localized atrophy of the inferior cervical spinal cord, asymmetrical flattening of the spinal cord and the loss of attachment between the posterior region of the dural sac and the subjacent lamina, displayed an accuracy level of over $80 \%$ in the identification of the disease [7]. The identification of a separation over $33 \%$ of the dural sac with its corresponding subjacent lamina is related to HD with a sensitivity and specificity rate of $93.5 \%$ and $98 \%$, respectively [7]. T2 sequences in axial plane visualized from C4 to C6 allows for better detection of this sign, as described in the study by Chen et al [7].

The increasing of the LDS and the widening of the posterior epidural space are adequately displayed in the MRI when obtained between 30 to $50^{\circ}$ of cervical angle. This is an essential condition for the diagnosis of HD. These findings seem to be a secondary outcome of the unbalanced bone development of the vertebral column and its content [2,3,7-11].

Different measurement values of LDS have been described between 3 to $9,8 \mathrm{~mm}$ and a mean of $6 \mathrm{~mm}[2,6,10,11]$. While other studies have reported a maximum displacement distance of 1.0 to $4.2 \mathrm{~mm}$ in healthy subjects compared to the distance between 6.1 to $7.8 \mathrm{~mm}$ in individuals diagnosed with HD [10]. The first patient in our case shows an LDS distance of $4.2 \mathrm{~mm}$ (Figure 1c) and the second patient has a LDS of $6 \mathrm{~mm}$ (Figure 2b).

The dynamic compression in flexion position over the arterial microvasculature of the anterior spinal cord horns produces hypoperfusion that resulted in ischemia. This is seen in the hyperintensity of the anterior horns displayed in the MRI -a.k.a, Snake Eyes Sign- [2,4,8-13] (Figure 1b)

'Snake Eyes Sign' is a radiological finding of focal myelomalacia of the anterior horns signaling late and irreversible damage of the medullary cord in HD. It is correlated with a bad prognosis of pyramidal signs and spinal cord atrophy, which indicates that it is not a benign, reversible disease, as described in the first studies published about the disease $[2,4]$.

Based on these hypotheses, to limit neck flexion is crucial in order to avoid the progression of the disease. The use of a cervical collar for the span of 3 to 4 years has been proven to be the least invasive treatment. An appropriate evolution and slow progression of the disease has been reported with this treatment $[2,11]$.

\section{Conclusion}

Although Hirayama Disease was initially described in Asian patients, recently it has been described in Western countries, where the compromise of the disease is found at a higher level (C5 and C6), as attested in our cases described on this paper [2]. Patient 1 presented atypical characteristics, including age and sudden onset of motor loss. The average age of the onset of the disease ranges between 15 to 25 years of age. This is due to the rapid growth that characterizes this stage of life $[2,4,11]$

In conclusion, despite its low frequency, HD must be considered as a differential diagnosis in young males with atrophy and strength loss predominantly in distal upper limbs. An active search of the signs by means of conventional and contrasted $\mathrm{MRI}$ in neutral and flexion position with an angle of at least 30 ㅇ45 을 is required for its early diagnosis and treatment. This will improve the prognosis of the disease. 


\section{References}

1. Hirayama K, Toyokura Y. TT. Juvenile muscular atrophy of unilateral upper extremity: A new clinical entity. Psychiatr Neurol Jpn. 1959; 61: 2190-2197.

2. Boruah DK, Prakash A, Gogoi BB, Yadav RR, Dhingani DD, et al. The importance of flexion MRI in Hirayama disease with special reference to laminodural space measurements. Am J Neuroradiol. 2018; 39: 974-980.

3. Huang YL, Chen CJ. Hirayama disease. Neuroimaging Clin N Am. 2011; 21: 939-950.

4. Xu H, Shao M, Zhang F, Nie C, Wang $H$, et al. Snake-Eyes Appearance on MRI Occurs during the Late Stage of Hirayama Disease and Indicates Poor Prognosis. Biomed Res Int. 2019; 2019: 1-8.

5. Gotkine M, Abraham A, Drory VE, Argov Z, Gomori JM, et al. Dynamic MRI testing of the cervical spine has prognostic significance in patients with progressive upper-limb distal weakness and atrophy. J Neurol Sci. 2014; 345: 168-171.

6. Lehman VT, Luetmer PH, Sorenson EJ, Carter RE, Gupta V, et al. Cervical spine MR imaging findings of patients with Hirayama disease in North America: A multisite study. Am J Neuroradiol. 2013; 34: 451-456.

7. Chen CJ, Hsu HL, Tseng YC, Lyu RK, Chen CM, et al. Hirayama Flexion Myelopathy: Neutral-Position MR Imaging Findings - Importance of Loss of Attachment. Radiology. 2004; 231: 39-44.
8. Desai JA, Melason M. Teaching Neurolmages: Anterior horn cell hyperintensity in Hirayama disease. Neurology. 2011; 77: 73.

9. Jakhere S, Wagh V. Hirayama's disease: The importance of flexion magnetic resonance imaging. J Postgr Med. 2011; 57: 4850 .

10. Lai V, Wong YC, Poon WL, Yuen MK, Fu YP, et al. Forward shifting of posterior dural sac during flexion cervical magnetic resonance imaging in Hirayama disease: An initial study on normal subjects compared to patients with Hirayama disease. Eur J Radiol. 2011; 80: 724-728.

11. Rosliakova A, Zakroyshchikova I, Bakulin I, Konovalov R, Kremneva $E$, et al. Hirayama disease: analysis of cases in Russia. Neurol Sci. 2019; 40: 105-112.

12. Chanson J B, Renaud M, Echaniz-Laguna A, Koob M. Dilation of epidural space and posterior soft tissue veins in Hirayama disease. BMJ Case Reports. BMJ Publishing Group. 2017; 2017.

13. Salome M, Barkhof F, Visser L. Hirayama disease; an atypical clinical manifestation of a cervical myelopathy with typical MRI features. BMJ Case Rep. 2017. 